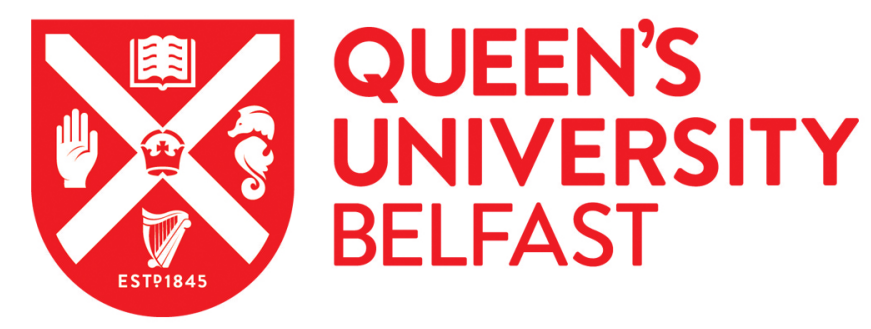

\title{
Characterization of a novel, short pulse laser-driven neutron source
}

Jung, D., Falk, K., Guler, N., Deppert, O., Devlin, M., Favalli, A., Fernandez, J. C., Gautier, D. C., Geissel, M., Haight, R., Hamilton, C. E., Hegelich, B. M., Johnson, R. P., Merrill, F., Schaumann, G., Schoenberg, K., Schollmeier, M., Shimada, T., Taddeucci, T., ... Roth, M. (2013). Characterization of a novel, short pulse laserdriven neutron source. Physics of Plasmas, 20(5), [056706]. https://doi.org/10.1063/1.4804640

\author{
Published in: \\ Physics of Plasmas
}

Document Version:

Publisher's PDF, also known as Version of record

Queen's University Belfast - Research Portal:

Link to publication record in Queen's University Belfast Research Portal

Publisher rights

Copyright VC 2013 AIP Publishing LLC. This work is made available online in accordance with the publisher's policies. Please refer to any applicable terms of use of the publisher.

\section{General rights}

Copyright for the publications made accessible via the Queen's University Belfast Research Portal is retained by the author(s) and / or other copyright owners and it is a condition of accessing these publications that users recognise and abide by the legal requirements associated with these rights.

Take down policy

The Research Portal is Queen's institutional repository that provides access to Queen's research output. Every effort has been made to ensure that content in the Research Portal does not infringe any person's rights, or applicable UK laws. If you discover content in the Research Portal that you believe breaches copyright or violates any law, please contact openaccess@qub.ac.uk. 


\section{Characterization of a novel, short pulse laser-driven neutron source}

D. Jung', K. Falk, N. Guler, O. Deppert, M. Devlin, A. Favalli, J. C. Fernandez, D. C. Gautier, M. Geissel, R. Haight, C. E. Hamilton, B. M. Hegelich, R. P. Johnson, F. Merrill, G. Schaumann, K. Schoenberg, M.

Schollmeier, T. Shimada, T. Taddeucci, J. L. Tybo, S. A. Wender, C. H. Wilde, G. A. Wurden, and M. Roth'

Citation: Physics of Plasmas 20, 056706 (2013); doi: 10.1063/1.4804640

View online: http://dx.doi.org/10.1063/1.4804640

View Table of Contents: http://aip.scitation.org/toc/php/20/5

Published by the American Institute of Physics

\section{Articles you may be interested in}

Neutron imaging with the short-pulse laser driven neutron source at the Trident laser facility

Journal of Applied Physics 120, 154901 (2016); 10.1063/1.4964248

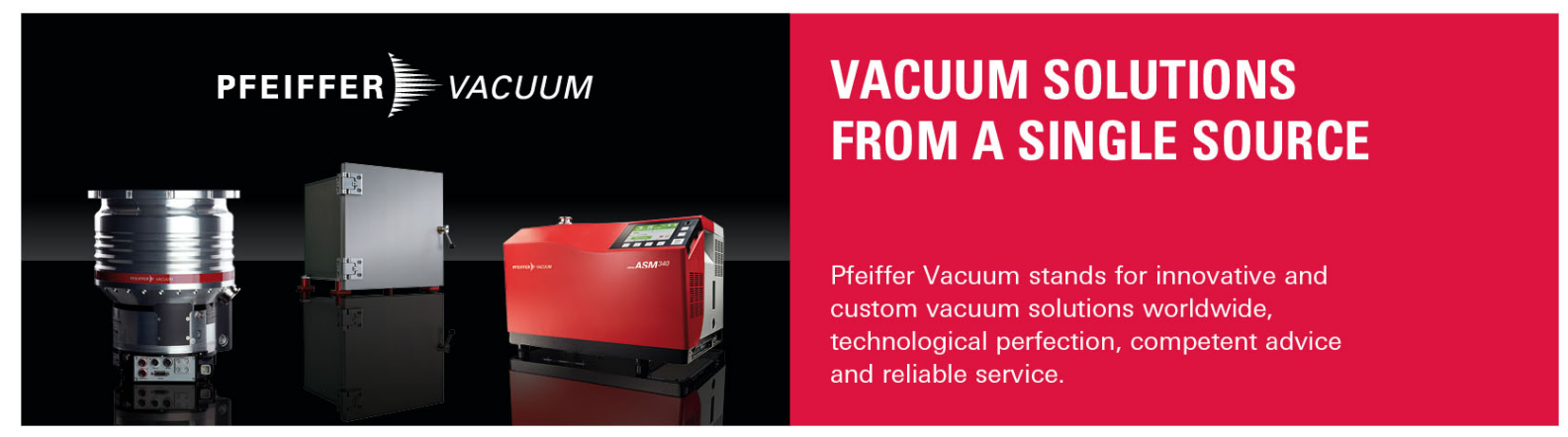




\title{
Characterization of a novel, short pulse laser-driven neutron source ${ }^{\text {a) }}$
}

\author{
D. Jung, ${ }^{1, b)}$ K. Falk, ${ }^{1}$ N. Guler, ${ }^{1}$ O. Deppert, ${ }^{2}$ M. Devlin, ${ }^{1}$ A. Favalli, ${ }^{1}$ J. C. Fernandez, ${ }^{1}$ \\ D. C. Gautier, ${ }^{1}$ M. Geissel,${ }^{3}$ R. Haight, ${ }^{1}$ C. E. Hamilton, ${ }^{1}$ B. M. Hegelich, ${ }^{1}$ R. P. Johnson, ${ }^{1}$ \\ F. Merrill, ${ }^{1}$ G. Schaumann, ${ }^{2}$ K. Schoenberg, ${ }^{1}$ M. Schollmeier, ${ }^{3}$ T. Shimada,${ }^{1}$ T. Taddeucci, ${ }^{1}$ \\ J. L. Tybo, ${ }^{1}$ S. A. Wender, ${ }^{1}$ C. H. Wilde, ${ }^{1}$ G. A. Wurden, ${ }^{1}$ and M. Roth ${ }^{2,1, c)}$ \\ ${ }^{1}$ Los Alamos National Laboratory, Los Alamos, New Mexico 87545, USA \\ ${ }^{2}$ Technische Universität Darmstadt, 64289 Darmstadt, Germany \\ ${ }^{3}$ Sandia National Laboratories, Albuquerque, New Mexico 87185, USA
}

(Received 17 January 2013; accepted 21 March 2013; published online 15 May 2013)

\begin{abstract}
We present a full characterization of a short pulse laser-driven neutron source. Neutrons are produced by nuclear reactions of laser-driven ions deposited in a secondary target. The emission of neutrons is a superposition of an isotropic component into $4 \pi$ and a forward directed, jet-like contribution, with energies ranging up to $80 \mathrm{MeV}$. A maximum flux of $4.4 \times 10^{9}$ neutrons/sr has been observed and used for fast neutron radiography. On-shot characterization of the ion driver and neutron beam has been done with a variety of different diagnostics, including particle detectors, nuclear reaction, and time-of-flight methods. The results are of great value for future optimization of this novel technique and implementation in advanced applications. ( 2013 AIP Publishing LLC. [http://dx.doi.org/10.1063/1.4804640]
\end{abstract}

\section{INTRODUCTION}

Neutrons can be used to probe and alter objects in a very different way than charged particles or electro-magnetic radiation. As the interaction is very different, complementary information can be obtained, so that over the last decades neutron research has spread from nuclear physics into fields like biology, ${ }^{1}$ archeology, ${ }^{2}$ medicine, material science, and high energy density physics. ${ }^{3,4}$ Neutrons are conventionally generated either in nuclear reactors for continuous flux or using particle accelerators for pulsed beams. Based on their size, it is clear that access to intense beams is so far limited to large scale facilities and there has been a growing need for compact and at the same time intense sources over the recent years. ${ }^{5}$ For more than a decade, intense short pulse lasers have been used as a novel source of energetic ion beams. ${ }^{6-12}$ After many years of research, the main characteristics of these ion beams can be summarized as: (a) high particle numbers of up to $10^{13}$ ions per laser pulse $;^{6}$ (b) energies up to several tens of $\mathrm{MeV} / \mathrm{amu}$ in an exponentially decaying energy distribution; ${ }^{10,13}$ (c) excellent beam quality; ${ }^{14}$ and (d) the possibility of shaping and tailoring the beam using structured targets and shaped laser intensity distributions. ${ }^{11,15}$ The most widely investigated mechanism so far has been the Target Normal Sheath Acceleration (TNSA) ${ }^{6-8}$ mechanism. It is based on the onset of huge quasi electro-static fields to accelerate ions from solid, thin foil targets when the laser drives the electrons through the solid material and off the targets rear surface. According to the nature of this mechanism, all ions at the rear surface are field ionized and then accelerated with respect to their charge to mass ratio. Due to always present, hydrogenous contamination layers at the target surface, most of the experiments have resulted in proton beams,

\footnotetext{
${ }^{a)}$ Paper YI2 4, Bull. Am. Phys. Soc. 57, 369 (2012).

${ }^{b)}$ Electronic mail: daniel.jung@outlook.com

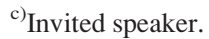

regardless of the target material. A more promising mechanism relies on the fact that a thin target, irradiated by a high contrast short pulse laser becomes relativistically transparent during the laser-plasma interaction, ${ }^{16}$ provided the right initial conditions are given. With on-target laser intensities exceeding $10^{18} \mathrm{~W} / \mathrm{cm}^{2}$, the relativistic mass increase of electrons by the Lorentz factor $\gamma$ can lead to a condition, where the plasma frequency $\omega_{p}$ drops below the laser frequency $\omega_{\lambda}$, so that the target is relativistically transparent to the laser light. When this happens, the laser can interact with the entire target volume and accelerate all ions contained within the laser-plasma interaction region to very high energies. This mechanism is known as the Break Out Afterburner (BOA) mechanism ${ }^{17,18}$ and has been experimentally verified at the LANL Trident laser facility. ${ }^{19,20}$ Here, the dependence on the charge to mass ratio $q / m$ is no longer the key parameter, and all target ions rather than only protons from surface contaminants can be efficiently accelerated without special target treatment. As all these experiments basically constitute a very bright and compact ion accelerator, there have been attempts to use them to produce a laser-driven neutron source. ${ }^{21-23}$ One of the most promising reactions to use is the combination of a fast deuteron beam and a light converter material with a high cross section for neutron generation as described by Petrov et al..$^{24}$ Recently, neutrons with maximum energies of $18 \mathrm{MeV}$ and a flux of $8 \times 10^{8}$ (neutrons)/sr have been reported ${ }^{25}$ using this technique with a lithium converter. Here, we present a full characterization of a laser-driven neutron source, with maximum neutron energies of $80 \mathrm{MeV}$ and flux of $4.4 \times 10^{9} \mathrm{n} / \mathrm{sr}$ per shot, exploiting better acceleration capabilities of deuterons in the $\mathrm{BOA}$ regime.

\section{EXPERIMENTAL SETUP}

The experiments were carried out at the Los Alamos National Laboratory Trident laser facility. The experimental setup is shown in Figure 1. An F/3 off-axis parabolic mirror 
a)

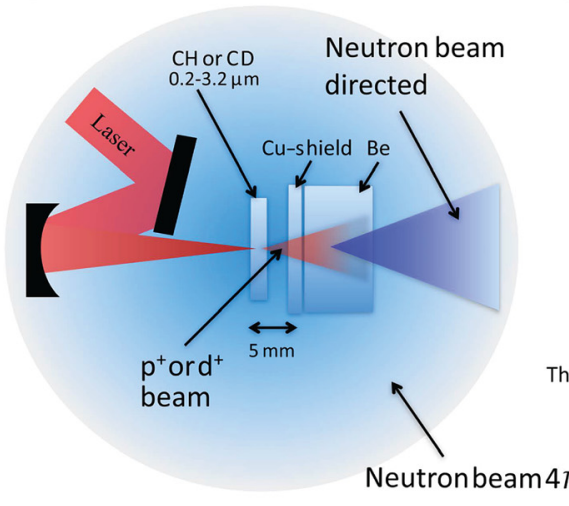

b)

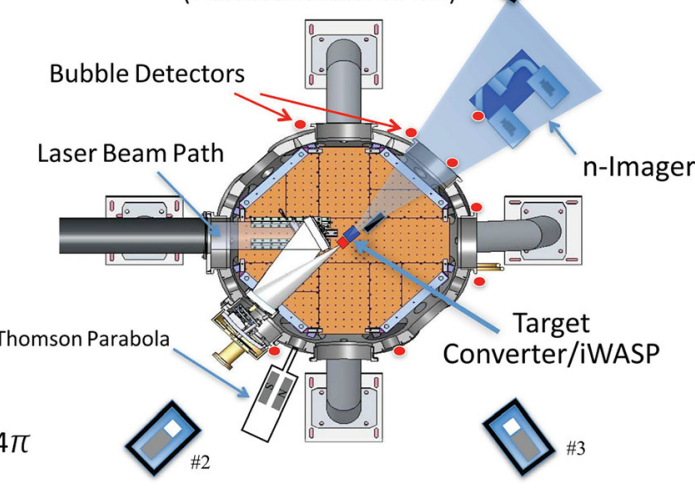

FIG. 1. (a) Experimental setup inside the target chamber. The laser is hitting a $\mathrm{CH}$ or CD foil and generates the driving proton or deuteron beam. This beam is deposited in a Be-converter on the laser propagation axis behind the target. (b) Global experimental setup. Around the target chamber, a variety of diagnostics for laser pulse and neutron beam characterization are placed. For the latter nTOF, spectrometers consisting of a plastic scintillator coupled to PMT, bubble detectors, and a neutron-imager are used. Not shown is the iWASP that has been used to analyze the driving ion right beam behind the target prior to the actual experiment. is used to typically focus $80 \mathrm{~J}$ of $1.053 \mu \mathrm{m}$ laser light in a 600 fs pulse. The on-target focus has been measured to be $\sim 6 \mu \mathrm{m}$ in radius $\left(1 / \mathrm{e}^{2}\right.$-condition, containing $>60 \%$ of the laser energy) with a peak intensity of $5 \times 10^{20} \mathrm{~W} / \mathrm{cm}^{2}$. The laser pulse duration and beam parameters were carefully recorded during the whole campaign. Thin, free-standing, plastic $\left(\mathrm{CH}_{2}\right)$ and deuterized plastic $\left(\mathrm{CD}_{2}\right)$ foils with thicknesses from $200 \mathrm{~nm}$ to $3.2 \mu \mathrm{m}$ were used to generate proton and deuteron beams. The high temporal contrast of the Trident laser with $10^{-7}$ at -4 ps (ratio of preceding laser irradiation compared to the peak intensity) ${ }^{26}$ enables interaction of the laser with a highly overdense target even for nm-scaled targets ${ }^{16}$ and hence very efficient acceleration of deuterons through the BOA mechanism. The proton and deutron beams were deposited in a Beryllium converter, providing a high cross section for neutron production and at the same time minimizing generation of unwanted high-energy bremsstrahlungs photons.

\section{A. Ion beam diagnostics}

As the ion beam is the primary driver for the neutron generation, beam parameters are an important part of the experiment. Evaluation of the neutron generation performance requires detailed knowledge of the driver in terms of energy distribution, particle numbers and energy content, as well as beam divergence. We used several independent diagnostics to characterize the driving ion beam for these parameters. In this experiment, where ion acceleration is done in the BOA regime, critical beam parameters such as highenergy cutoffs, angular ion distribution, conversion efficiency, and ion species are very different from typical TNSA-generated ion beams. While in TNSA the ion beam is typically "Gaussian"-like distributed with peak energy and flux in the beam center, an ion beam generated in the BOA regime is shaped by the $2 \mathrm{D}$ and $3 \mathrm{D}$ dynamics during the relativistic transparent phase of the interaction governing the acceleration. One is the azimuthal symmetry break even for a symmetric laser profile; the symmetry break causes generation of ion lobes in the spectrum ${ }^{27}$ and hence maximum energies and also maximum densities to appear off-axis.

\section{1. iWASP spectrometer}

Here, we use the ion wide angle spectrometer (iWASP) as described in Ref. 28. The iWASP measures the ion beam angularly resolved over approximately $20^{\circ}$ in a plane perpendicular or parallel to the laser polarization axes. It is based on a strong, large, magnetic field generated by a wedged yoke perpendicular to the ion propagation direction. The field introduces an energy dependent dispersion of the particle beam that is entering the iWASP through a long slit positioned parallel to and in front of the magnetic field. With a slit of $\sim 20 \mu \mathrm{m}$ width and $1 \mathrm{~cm}$ length, the spectrometer covers a solid angle of $\sim 4 \times 10^{-1} \mathrm{msr}$; this is 3-5 orders of magnitude higher than in typical Thomson parabolas. ${ }^{29}$ The high solid angle captured, and hence the high fraction of the beam that is analyzed ensures highest accuracy in measuring all important beam parameters. This is done simultaneously for $\mathrm{C}^{6+}$ and $\mathrm{H}^{+}$(and $\mathrm{D}^{+}$) from $\mathrm{CH}_{x}$ (and $\mathrm{CD}$ ) targets and is accomplished by taking advantage of the hugely different stopping power of each of the particles in a stacked detector in the iWASP. The detector consists of a $25 \mu \mathrm{m} \mathrm{Al}$ layer as a shield for direct and stray laser light, a CR39 nuclear track detector, ${ }^{30,31}$ and an image plate. ${ }^{32}$ The $1 \mathrm{~mm}$ thick CR39 mainly detects carbon ions; it is transparent to protons and deuterons above $11 \mathrm{MeV}$ and $15 \mathrm{MeV}$, respectively. The BAS-TR image plate behind the CR39 detects protons and deuterons above these energies (see Ref. 28 for details). The energy resolution of the iWASP is better than $10 \%$ at energies of $50 \mathrm{MeV} / \mathrm{amu}$. Fig. 2 shows a typical angularly resolved deuteron spectrum measured with the iWASP; the spectrum has been taken during a shot on a $530 \mathrm{~nm}$ thick CD foil target at Trident. A Thomson parabola was additionally measuring ion energies in front of the target (see Fig. 1(b)) in correlation to the iWASP measurements. For shots, where the converter was in place and the iWASP could not be used, the Thomson parabola measurements served as a rough reference for ion energies.

\section{Nuclear activation-based imaging spectroscopy (NAIS)}

While the iWASP is able to simultaneously measure ion spectra for different species angularly resolved, it does it only for the fraction of the beam that is actually entering the slit in front of the iWASP. For an analysis of the beam shape itself, we use a NAIS technique. ${ }^{33}$ This technique is similar to the well-known radio-chromic film imaging spectroscopy (RIS) ${ }^{34}$ but does not suffer from saturation effects and is 


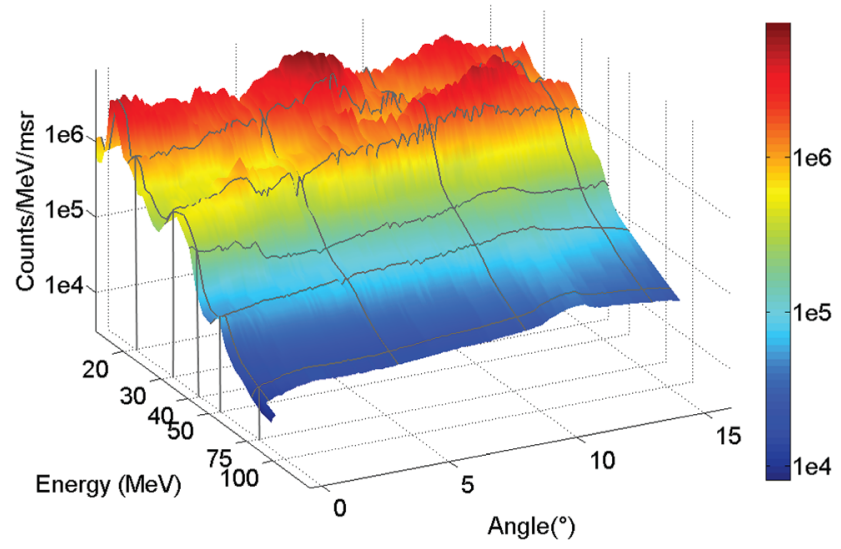

FIG. 2. Angularly resolved deuteron spectrum from $-0.5^{\circ}$ to $16.5^{\circ}$, measured in a plane parallel to the laser polarization axes. $0^{\circ}$ is laser and target normal. The flux is color coded in PSL/MeV/msr.

reusable. The NAIS technique uses a stack of several copper foils $(50 \mu \mathrm{m}-250 \mu \mathrm{m}$ thickness), which in this experiment were placed around the entrance slit of the iWASP. Energetic protons and deuterons above the neutron separation threshold of $4 \mathrm{MeV}$ cause a transmutation of copper into zinc via ${ }^{63} \mathrm{Cu}(\mathrm{p}, \mathrm{n}){ }^{63} \mathrm{Zn}$; hence, NAIS cannot be used for any other ion species. The reaction cross section peaks at a proton energy of $10 \mathrm{MeV}$, and decreases rapidly until around $15 \mathrm{MeV}$ the cross section for the ${ }^{63} \mathrm{Cu}(\mathrm{p}, 2 \mathrm{n}){ }^{63} \mathrm{Zn}$ reaction increases rapidly. The unstable $\mathrm{Zn}$-isotope quickly decays back into copper with a very convenient half-life of $38 \mathrm{~min}$ emitting a positron, which can be detected easily with an image plate based auto-radiography. Using post-shot gamma spectroscopy, the individual reactions can be separated and thus a rough energy spectrum can be derived. In each of the thin copper sheath-when exposed to a proton beam from a short pulse laser plasma-nuclei are activated according to the energy spectrum convoluted with the different cross sections. Each copper layer represents a single energy bin. The energy of the bin is given by the position of the layer in the stack, taking the known dielectronic stopping power of protons in copper into account. The width of the bin is given by the thickness of the specific layer. The last layer with a measurable activation signal gives the maximum proton energy minus the $4 \mathrm{MeV}$ activation threshold. The more layers used in the stack, the more detailed the information about the spatial profile, the energy spectrum, and absolute particle numbers of the laser-accelerated proton beam. Fig. 3 shows an on-contact auto-radiography of activated copper plates from a typical shot; it provides excellent spatial information for the specific energy bins of the copper stack. The NAIS in combination with the iWASP provides an excellent tool for analyzing the proton/deuteron beam, which acts as the neutron-driver.

\section{B. Neutron diagnostics}

\section{Nuclear activation}

Copper activation can also be used for neutron detection via $(n, 2 n)$-reactions. Here, a 2 in. diameter and 0.25 in. thick copper disk (114.7 g) was used as a neutron activation sample and placed in various locations around (or inside) the Trident target chamber. The copper samples are $99.99 \%$ pure and naturally composed of ${ }^{63} \mathrm{Cu}(69.2 \%)$ and ${ }^{65} \mathrm{Cu}(30.8 \%)$. Since copper activation can also be done with $(\mathrm{p}, \mathrm{n})$ reactions as for the NAIS technique, it is important to make sure that fast protons do not interact with the sample. The chamber walls are very efficient in blocking all protons; however, when placed inside the chamber, a location outside of the ion beam is necessary to achieve only $(n, 2 n)$ activation of the copper. The ${ }^{63} \mathrm{Cu}(\mathrm{n}, 2 \mathrm{n}){ }^{62} \mathrm{Cu}(\beta+)$ reaction has an energy threshold of $11 \mathrm{MeV}$ (cross section of $454 \mathrm{mb}$ for $14 \mathrm{MeV}$ neutrons ${ }^{35}$ ). ${ }^{62} \mathrm{Cu}$ decays with a half life of $9.74 \mathrm{~min}$ and emits a positron resulting in the production of two $511 \mathrm{keV}$ gamma rays upon annihilation. For the copper disk size we used, this corresponds to about 3 atoms activated for every neutron $/ \mathrm{cm}^{2}$ fluence through the disk. The annihilation radiation was detected with a pair of back to back 3 in. diameter $\mathrm{NaI}(\mathrm{Tl})$ scintillation crystals coupled to photo-multipliers, gated in coincidence (with a background of less than 1 count $/ \mathrm{min})$. They have an overall system efficiency of $\sim 12 \%$ (determined using a ${ }^{68} \mathrm{Ge}$ positron source) to count positrons from the sample. A second reaction which occurs is ${ }^{65} \mathrm{Cu}(\mathrm{n}, 2 \mathrm{n}){ }^{64} \mathrm{Cu}(\beta+)$ with a $10 \mathrm{MeV}$ threshold, and cross section of $906 \mathrm{mb}$ at $14 \mathrm{MeV}$; it decays with a half life of 12.7 h. By plotting the combined decay rates, taken with 1min counting windows, the two reactions can easily be

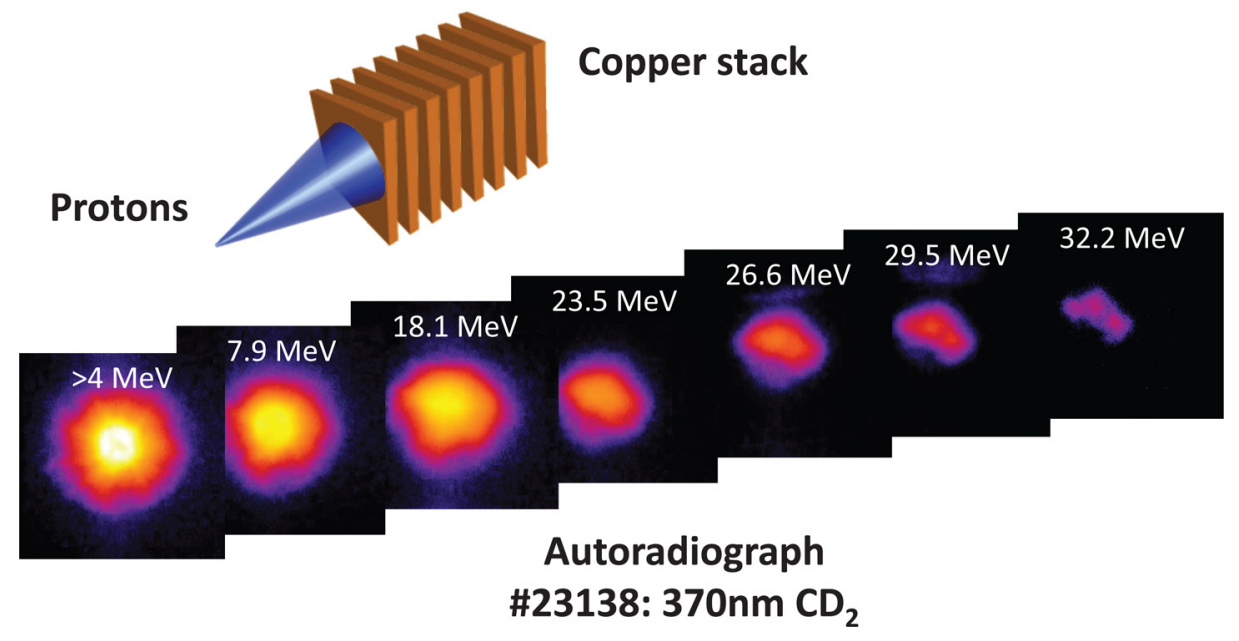

FIG. 3. Schematic depiction of the nuclear activation-based imaging spectroscopy. The black squares show auto-radiography scans of different layers of the copper stack. Each layer corresponds to small energy window, depending on the position of the layer on the stack and its thickness. The center energy is noted in the top of each square. Brighter colors correspond to higher signal. 
separated. The copper sample was typically placed outside of the Trident target chamber flange, $90^{\circ}$ off-axis with respect to the beam propagation direction, and $87 \mathrm{~cm}$ from the target foil. With the activation measurements, the number of neutrons that went through the copper sample can be calculated. The initial number of activated atoms $N_{0}$ in copper sample right after the shot is given by $N(t)=N_{0}(1-\exp (-\Delta t / \tau))$, where $\Delta t=1 \mathrm{~min}$ and the half life $\tau=9.7 \mathrm{~min}$. Taking into account the sample size, nuclear cross section, detector efficiency, and gamma ray absorption within the copper, the measured number of initially activated copper atoms translates into the number of neutrons per unit area for the sample.

\section{Bubble detectors}

A more direct technique employed to measure the neutron yield makes use of commercially available bubble detectors (BTI). ${ }^{36}$ The bubble detectors have been used to measure the spatial dependence of the integrated neutron yield (in time and energy across the projected area of the bubble detector). A significant advantage of the bubble detectors is their sensitivity to a wide range of neutron energies from less than $1 \mathrm{MeV}$ to up to $100 \mathrm{~s}$ of $\mathrm{MeV}$. The detectors are made of super-heated droplets dispersed in an elastic gel medium such as Freon; typically, there are tens of thousands of these small $(10 \mu \mathrm{m}$ diameter) droplets in an $8 \mathrm{ml}$ polycarbonate tube. They are stable at normal temperatures and are completely insensitive to gamma radiation, as the energy deposition of electrons generated from high energy photons penetrating the detector is too low. This makes them an ideal candidate for laser-plasma experiments; especially at high power laser systems such as Trident, where radiation background from the interaction can cause a significant decrease in the signal to noise ratio. Neutrons, on the other hand, causing hard knock-on reactions, will excite recoil ions, which can transfer enough energy to vaporize a droplet and form a bubble with a much larger volume. These bubbles are easily observable with the naked eye or a computerized readout system; the latter has been used throughout this experiment. If not used, the detectors are stored under pressurized conditions to prevent spontaneous bubble formation; prior to the experiment, the detector is armed by releasing the pressure. Detectors with sensitivities from 7 bubbles/ mrem up to 62 bubbles/mrem have been used to achieve unsaturated, but statistically significant signal depending on the expected neutron flux. In order to measure the angular distribution of the laser-generated neutrons, a total of up to 12 detectors was placed in different positions and distances around the point-like source. To obtain the neutron yield from the bubble detector measurements at each position, the response function for these detectors is used. The function is shown in Fig. 4; blue stars represent experimental data from Refs. 37 and 38. In contrast to the above mentioned nuclear activation of copper, bubble detectors are sensitive to neutrons below $1 \mathrm{MeV}$. The response is quite flat for neutron energies up to $30 \mathrm{MeV}$, but for higher energies, the sensitivity is dropping by almost a factor of 3. Since the experiment showed neutron energies of up to $80 \mathrm{MeV}$, we used the spectral distribution of the neutron beam extracted from the

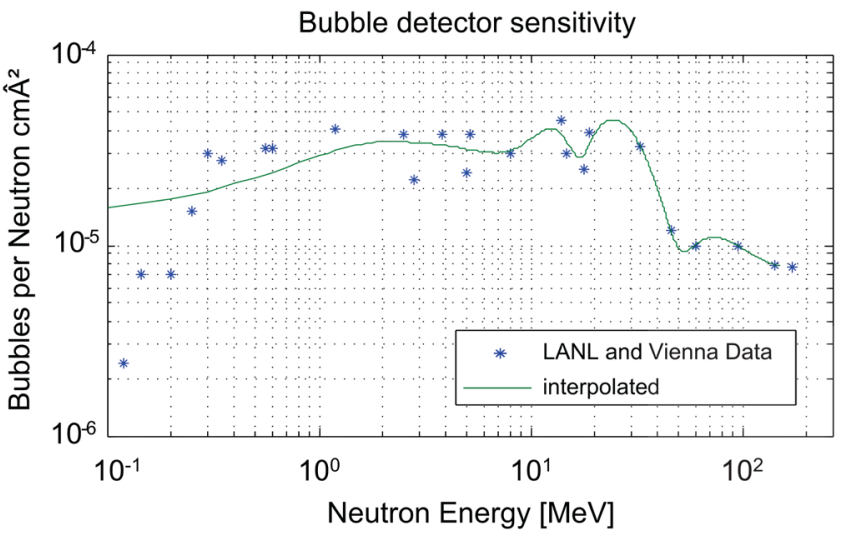

FIG. 4. Sensitivity of BTI-BND bubble detector units ( 1 bubble/mrem calibration) for different neutron energies. Experimental data are derived from Refs. 37 and 38. The sensitivity is reduced by more than a factor of three for neutron energies exceeding $30 \mathrm{MeV}$.

neutron Time-of-Flight (nToF) detector measurements (see below) to obtain a more accurate value for the neutron flux. The convolution of the interpolated bubble detector sensitivity (green solid line) with the neutron spectrum from the nTOF measurements and the distance of the bubble detectors to the source give the total neutron yield in neutrons/sr. After each measurement, the detectors are pressurized again, which compresses the bubbles back into their liquid droplet form allowing to reuse them.

\section{3. nTOF detectors}

The neutron energy spectrum was measured in different directions using several nTOF detectors $(10 \mathrm{~cm}$ diameter, $1.88 \mathrm{~cm}$ thick NE102 plastic scintillators coupled to fast $12.5 \mathrm{~cm}$ Photo Multiplier Tubes, PMTs). Three detectors were used as illustrated in Fig. 1(b): Detector \#1 was placed at $15^{\circ}$ with respect to the laser forward propagationdirection, $327 \mathrm{~cm}$ from the driver target. Detector \#2 was placed at $10^{\circ}$ with a distance of $228 \mathrm{~cm}$. It was later placed at $160^{\circ}$ with respect to the laser propagation direction, at a distance of $335 \mathrm{~cm}$ and finally of $569 \mathrm{~cm}$ from the driver target. Detector \#3 was placed at $90^{\circ}$ with respect to the laser propagation direction, monitoring the neutron spectrum perpendicular to the neutron beam path at a distance of $273 \mathrm{~cm}$ (see Fig. 1(b)). During the campaign, the nTOF detectors were shielded against $\mathrm{x}$-rays with increasing numbers of lead bricks up to $25 \mathrm{~cm}$ to minimize the prompt gamma signal and enhance the signal to noise ratio for high energy neutrons with a shorter time of flight. The detection limit for high energy neutrons is given by the overlap of the signal with the gamma flash. Due to space constrains, the detectors could not be moved further away in the experiment, giving a detection limit of $\sim 200 \mathrm{MeV}$ for this campaign. Each nTOF detector signal was recorded by a fast digital oscilloscope; a typical measurement is shown in Fig. 5 (blue solid line). The strong, prompt signal from the laser driven $\mathrm{x}$ - and $\gamma$-rays (green solid line) and the known signal delay inside the PMT serve as a time reference for the neutron spectrum analysis. The energy dependent response function of the spectrometers is calculated by convolution of MCNP-PoliMi data for the 


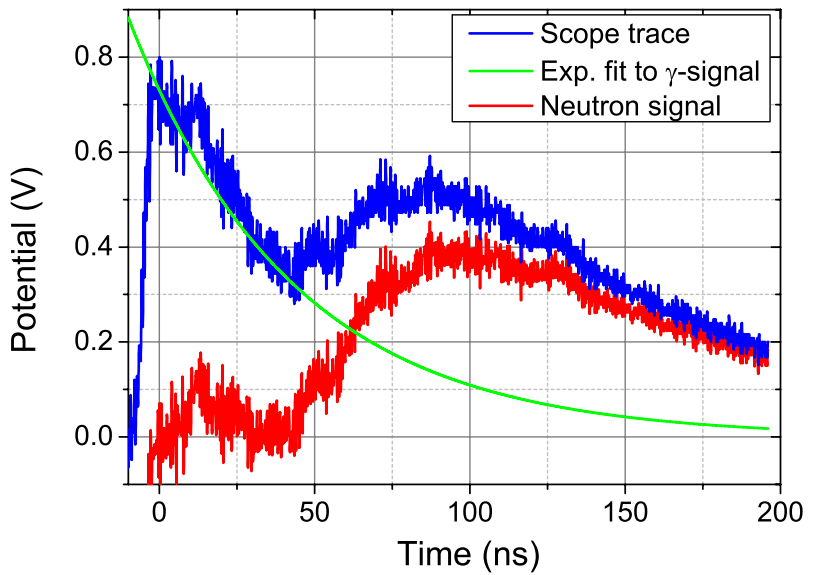

FIG. 5. Typical nTOF signal recorded with a fast digital oscilloscope. The blue solid line is the raw scope trace. The red solid line shows the raw neutron spectrum, accounting for the gamma flash, which serves as time reference (the green solid line).

scintillator material and the neutron energy loss due to the lead shielding in front of the detectors. Fig. 6 shows the quite flat response function of the nTOF detectors from $0.5 \mathrm{MeV}$ up to $50 \mathrm{MeV}$ for different lead shielding.

\section{Neutron imager}

Neutrons emitted into the forward direction (the propagation direction of the laser and the incident ion beam) were in addition observed using an imaging neutron detector. The neutron imager is a bundled array of scintillating fibers (BCF-20) connected to a fast gated micro channel plate (MCP) followed by a fiber rope and a high resolution CCD camera. This system ${ }^{39,40}$ was developed at LANL initially to diagnose the inertial confinement fusion experiments at the National Ignition Facility (NIF) and used extensively at the OMEGA laser facility. Neutrons, incident into the $5 \mathrm{~cm}$ thick fiber array of the detector (consisting of $500 \mu \mathrm{m}$ diameter fibers), generate light that is transported through the fibers, collimated by a coherent fiber taper, amplified by a gated micro channel plate, and finally detected in a cooled, highresolution CCD camera. The scintillator is also sensitive to the gamma rays produced during the initial laser-target interaction. The fast decay constant (1/e) of the scintillator is

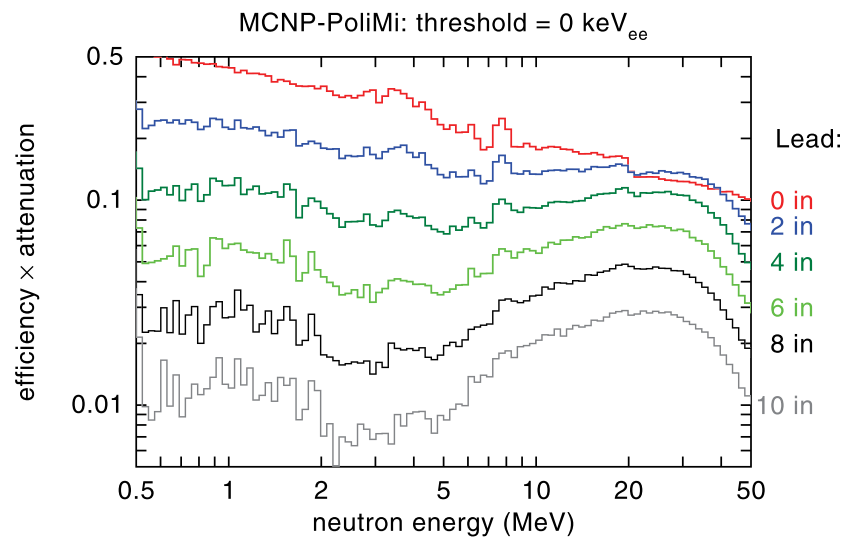

FIG. 6. Spectral response of the nTOF detectors as a function of the neutron energy for different thicknesses of lead shielding.
$2.7 \mathrm{~ns}$, so that the gamma contribution can be eliminated from the signal created by the neutrons that arrive $20 \mathrm{~ns}$ or later after the gamma flash. This requires an appropriate timing of the MCP gate in addition to a $2 \mathrm{~cm} \mathrm{~Pb}$ shielding in front of the scintillator. The system is able to use the gamma flash itself or the neutrons in any selected energy range for radiography or imaging purposes. The latter is of particular interest to radiography applications, as it allows to study material properties using different neutron energies. During the experiment, the neutron imager was placed $2 \mathrm{~m}$ behind the main laser-plasma interaction region, almost centered to the laser and ion beam propagation direction (see Fig. 1(b)). Different materials were used in front of the imaging system to investigate attenuation of the neutrons and the neutron imaging quality. To enhance the edge contrast of the image, the source size was decreased from a square of $50 \times 50 \mathrm{~mm}$ to circle of $3 \mathrm{~mm}$ diameter by reducing the distance of the primary ion source to the converter target and adjusting the dimensions of the converter to the energy distribution and shape of the driving ion beam. A flat-field reference image for the neutron imager was recorded using a neutron source with diameter of $>50 \mathrm{~cm}$. The source was generated by removing the beryllium converter and dumping the driving ion beam into the steel target chamber wall, creating neutrons from $(\mathrm{p}, \mathrm{n})$-reactions and deuteron breakup. A large CR39 nuclear track detector plate served as a calibration reference to the neutron imager. Therefore, the CR39 was fielded directly in front of the imager; as it is insensitive to $\mathrm{x}$-rays and electrons and no ions are to be expected to pass through the chamber wall, the CR39 records a clean neutron reference signal. A typical neutron image of an arrangement of tungsten blocks is shown in Fig. 7. Detailed results of the neutron imager will be published elsewhere.

\section{RESULTS}

In the beginning of the experiment, the proton and deuteron beam driving the neutron generation was analyzed. The driver beams are generated by irradiation of $\mathrm{CH}$ and $\mathrm{CD}$ targets with laser peak-intensities of $5 \times 10^{20} \mathrm{~W} / \mathrm{cm}^{2}$. Target thicknesses range from $100 \mathrm{~nm}$ up to $3.2 \mu \mathrm{m}$ and allow ion

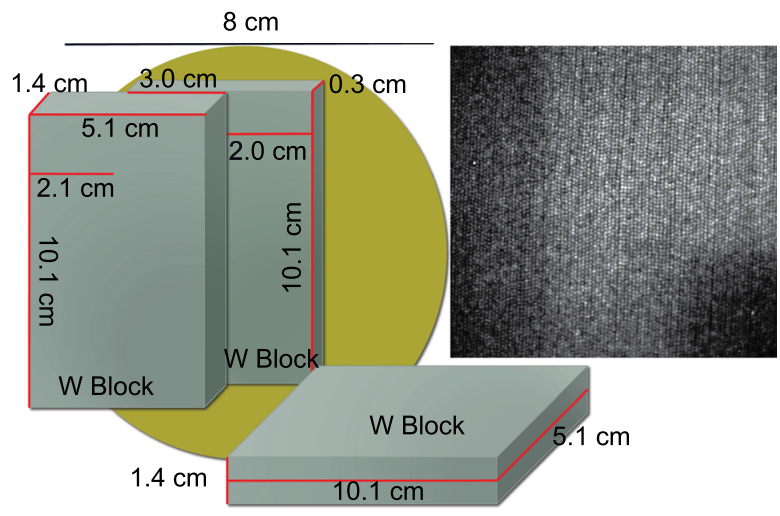

FIG. 7. Neutron image of an arrangement of 3 tungsten blocks of different dimensions as measured with the scintillator in the neutron imager. The imager started to record $31 \mathrm{~ns}$ after the $\mathrm{x}$-ray/gamma flash. The gate time of $80 \mathrm{~ns}$ made the detector sensitive to neutron energies between $2.5 \mathrm{MeV}$ and $15 \mathrm{MeV}$. Brighter grey scales correspond to higher neutron signal. 
acceleration in the before mentioned Break-Out-Afterburner regime. In BOA, the laser energy is dominantly transferred to the ions during the overdense but relativistically transparent phase of the laser-plasma interaction. ${ }^{19,41}$ The target is relativistically transparent, when $N / \gamma \leq 1<N$ with $N$ $\equiv n_{e} / n_{c r}$ the normalized target electron density and $n_{c r}$ $=m_{e} \omega_{0}^{2} /\left(4 \pi e^{2}\right)$ the critical electron density above which the non-relativistic plasma is opaque to laser light of frequency $\omega_{0}$. During this phase, efficient acceleration of all target ions to very high energies takes place; electrons, driven by the laser, co-move with the ionized target and transfer energy to the ions; while at same time, the laser continuously transfers energy to the electrons. ${ }^{17}$ When the target is relativistically transparent at the time the peak of the laser pulse is interacting with the target, the mechanism works most efficiently.

As a result, all ions are accelerated to high energies independent of their $\mathrm{q} / \mathrm{m}$ ratio. ${ }^{18}$ Using a deuteron-rich target, such as a $C D_{2}$ plastic target where almost all protons are replaced by deuterons, can thus be used to efficiently accelerate deuterons in the BOA regime. This is in stark contrast to TNSA, where electron heating in the laser field and energy transfer to the ions is much less coupled and ions, predominantly protons, are accelerated only from the target surface, rendering TNSA a rather inefficient mechanism to accelerate deuterons. Optimum acceleration with BOA depends on the actual laser intensity and contrast and the target density and thickness; all these parameters influence the evolution of the electron density during the laser-plasma interaction and hence the timing of a relativistically transparent phase. During this particular experiment, the optimum target thickness was approximately $400 \mathrm{~nm}$ for the $\mathrm{CH}$ and $\mathrm{CD}$ targets at which peak energies of $80 \mathrm{MeV}$ and $100 \mathrm{MeV}$ have been measured for protons and deuterons, respectively. With thinner or thicker targets, particle energies and numbers drop rapidly (for more details, see Ref. 19). The spectra were recorded with the iWASP, which was placed at a distance approximately $25 \mathrm{~mm}$ behind the target (without the converter in place). A typical deuteron spectrum is shown in Fig. 8; the spectrum is an averaged line-out from $6^{\circ}$ to $8^{\circ}$ of

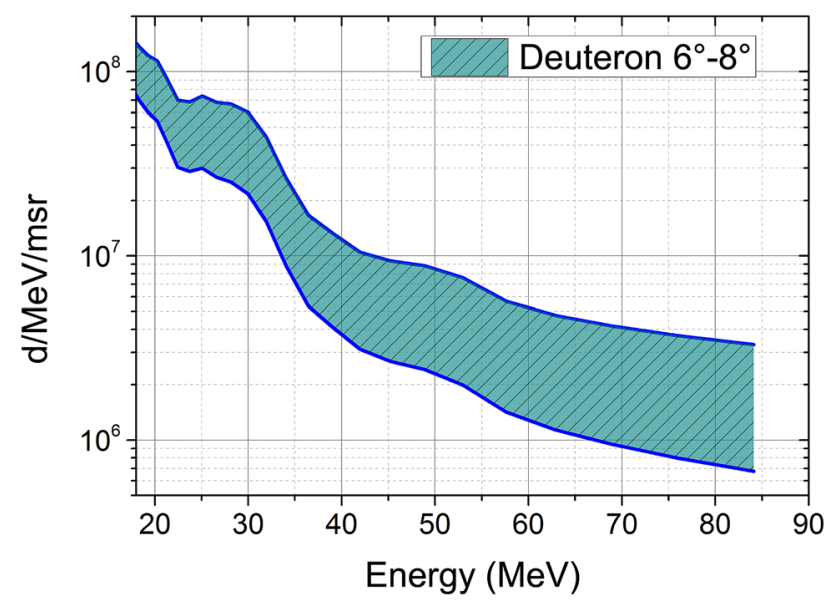

FIG. 8. Typical deuteron spectrum from a $400 \mathrm{~nm}$ CD foil target. The spectrum is an average from $6^{\circ}$ to $8^{\circ}$ of an angularly resolved measurement such as shown in Fig. 2. Numbers are approximated based on image plate calibrations published in Refs. 42 and 43. the angularly resolved 2D-map shown in Fig. 2. From the iWASP measurements, we approximate the number of protons with $E>11 \mathrm{MeV}$ and deuterons $E>15 \mathrm{MeV}$ (the lower detection threshold of the iWASP) within a $15^{\circ}$ cone to $(1 \pm 0.5) \times 10^{11}$ and $(5 \pm 3) \times 10^{11}$, respectively. For this approximation, we assumed a radially symmetric beam distribution and took the IP calibrations from Refs. 42 and 43. This would give a conversion efficiency of laser light into deuterons on the order of $0.5 \%$.

In the main part of the experiment, these beams were dumped in a beryllium converter. The converter was placed at distances of $5 \mathrm{~mm}$ up to $5 \mathrm{~cm}$ behind the driver target to control the size of the neutron source. The lateral dimensions of the converter were adjusted accordingly to capture the full ion beam, which had an average cone half-angle of about $30^{\circ}$ (see Ref. 19); the length of the converter was typically $5 \mathrm{~cm}$, sufficiently long to stop protons and deuterons with energies of $100 \mathrm{MeV}$ and $135 \mathrm{MeV}$, respectively. It should be noted that with the Be-converter in place, the use of the iWASP ion diagnostic was not possible. For these shots, the Thomson parabola at the front side of the target was used as a reference for ion energies (see Fig. 1(b)). The measurements indicate that the shot to shot variations of maximum energies of the driving beam are minimal and fluctuate around the values measured with iWASP. Beryllium constitutes an excellent converter as it has a high cross-section for neutron generation and at the same time has a low x-ray yield from electron and ion deposition in comparison to high- $\mathrm{Z}$ materials. In the converter, neutrons are expected to be produced by two dominant mechanisms, depending on the species and energy of the driver beam. With a pure proton beam, neutrons should mostly be generated in ${ }^{9} \mathrm{Be}(\mathrm{p}, \mathrm{n}){ }^{9} \mathrm{~B}$-reactions; ${ }^{9} \mathrm{Be}(\mathrm{p}, 2 \mathrm{n}){ }^{8} \mathrm{Be}$ reactions can also contribute to the process at higher proton energies. ${ }^{44}$ Since the incident proton beam typically has an exponentially decaying energy spectrum and scattering of protons takes place in the converter, the resulting neutron distribution is expected to be quasi-isotropic, i.e., close to a homogeneous distribution in $4 \pi$. With a deuteron beam, neutrons can be generated in ${ }^{9} \mathrm{Be}(\mathrm{d}, \mathrm{n}){ }^{10} \mathrm{~B}$ and deuteron-breakup reactions, i.e., by splitting of the loosely bound deuteron into a proton and a neutron. The proton from the breakup-reaction can subsequently generate another neutron via a follow-on ${ }^{9} \mathrm{Be}(\mathrm{p}, \mathrm{n}){ }^{9} \mathrm{~B}$ or ${ }^{9} \mathrm{Be}(\mathrm{p}, 2 \mathrm{n})^{8} \mathrm{~B}$-reaction. In this case, the neutron distribution will have two components: the $(\mathrm{d}, \mathrm{n})$ and $(\mathrm{p}, \mathrm{n})$ reactions give a point-like source distribution as described before, and the breakup-reaction, on the other hand, is directed in the general direction of the driving deuteron beam. The cone of the directed part of the neutron beam depends on scattering of deuterons in the converter and the original divergence angle of the deuteron beam itself.

Starting with $\mathrm{CH}$ foils between $300 \mathrm{~nm}$ and $500 \mathrm{~nm}$ thickness - the optimum for BOA-proton beams with measured peak energies of up to $80 \mathrm{MeV}$ have been dumped in a beryllium converter. To prevent Be-contamination of the target chamber, the converter was shielded with layers of copper and plastic. The shielding sufficiently protected the converter from plasma blow-off and transmitted light. The 12 bubble detectors placed around the target chamber 


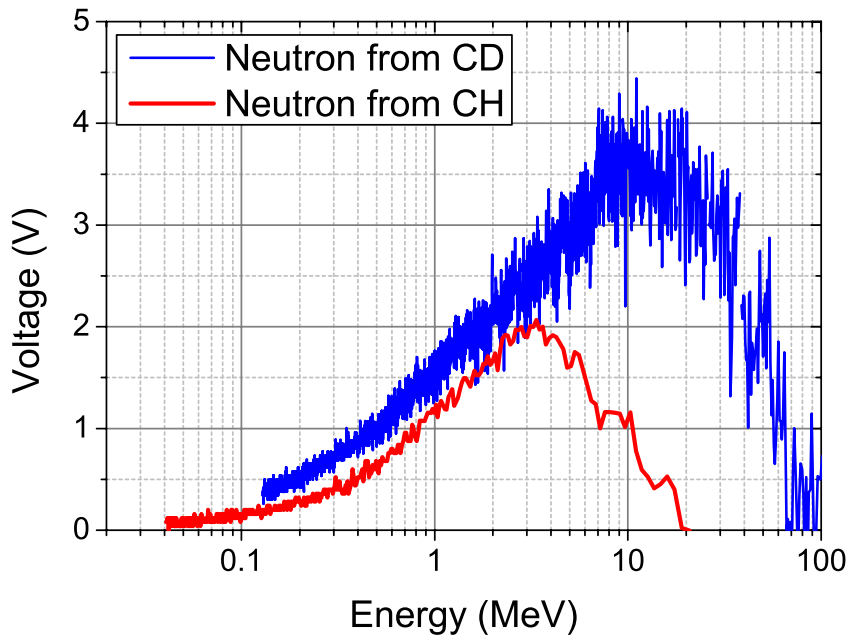

FIG. 9. Neutron spectra from a $400 \mathrm{~nm} \mathrm{CH}$ foil (red) and a $480 \mathrm{~nm}$ CD foil (blue), measured with an nTOF detector. Peak flux is around $4 \mathrm{MeV}$ with maximum neutron energies of $15 \mathrm{MeV}$ for the $\mathrm{CH}$ neutron spectrum. For the $\mathrm{CD}$ target, neutron flux peaks between $10 \mathrm{MeV}$ and $20 \mathrm{MeV}$ with maximum neutron energies of close to $100 \mathrm{MeV}$.

(see Fig. 1(b)) showed a nearly isotropic distribution, with an approximated neutron yield of up to $5 \times 10^{8} \mathrm{n} / \mathrm{sr}$. The nTOF detectors measured a peak neutron flux at an energy of around $4 \mathrm{MeV}$ and maximum neutron energies of $10 \mathrm{MeV}$ to $20 \mathrm{MeV}$. A typical spectrum is shown in Fig. 9 (red solid line). The results for $\mathrm{CH}$ targets are consistent with Ref. 25.

Changing to $\mathrm{CD}$ targets of the same thickness and without changing the laser parameters gave a driving beam of deuterons with measured energies of up to $100 \mathrm{MeV}$ and also protons from surface contamination and target impurities. The neutron beam distribution measured with the bubble detectors showed two components: an isotropic component from $(\mathrm{p}, \mathrm{n})$ and $(\mathrm{d}, \mathrm{n})$-reactions and a peaked forward component from deuteron breakup-reactions (see Fig. 10). The neutron yield in the forward direction was one order of magnitude higher with up to $4.4 \times 10^{9} \mathrm{n} / \mathrm{sr}$. All other directions measured much lower neutron fluxes of up to $1 \times 10^{9}$ $\mathrm{n} / \mathrm{sr}$. With an approximated deuteron number of $5 \times 10^{11}$ from the iWASP measurements, this gives a total conversion efficiency of $9 \times 10^{-3} \mathrm{n} / \mathrm{sr} /$ deuteron in the forward direction and $2 \times 10^{-3} \mathrm{n} / \mathrm{sr} /$ deuteron in $4 \pi$. These numbers are on the same order as predicted by Petrov et al. ${ }^{24}$ using a Lithium instead of a Beryllium converter with an average deuteron energy of $\sim 25 \mathrm{MeV}$.

Neutron numbers derived from the nuclear activation of copper placed orthogonal to the beam path were on the order of $5 \times 10^{8} \mathrm{n} / \mathrm{sr}$ for neutrons above the detection threshold of $11 \mathrm{MeV}$. This number is in good agreement with the $4 \pi$-flux measured by the bubble detectors that are sensitive to neutrons from below $1 \mathrm{MeV}$ to $>100 \mathrm{MeV}$. The source size has been estimated with a standard knife edge measurement. Here, a tungsten block in front of the neutron imager was used as edge. For the optimized beryllium converter with a $3 \mathrm{~mm}$ diameter, a source size of $2.5 \mathrm{~mm}$ FWHM was measured.

The nTOF-detectors detected the same forward directed neutron distribution as the bubble detectors. The ones placed orthogonal to the laser beam and parallel to its counterpropagation direction still measured the peak neutron flux at $4 \mathrm{MeV}$ with maximum energies of up to $20 \mathrm{MeV}$. However, in the direction of the driving ion beam, the neutron flux peaked at energies of up to $25 \mathrm{MeV}$ with maximum energies of $50 \mathrm{MeV}$ to $80 \mathrm{MeV}$. A typical spectrum is shown in Fig. 9 (blue solid line). The high maximum neutron energies imply that other neutron generating mechanisms are present such as pre-compound reactions. ${ }^{45}$ (Neutron velocities from the deuteron break-up are limited to the velocity of the original deuteron, i.e., not more than half of its energy.)

It should furthermore be noted that the forward directed neutron distribution vanishes and returns to a more $4 \pi$-like distribution, when $\mathrm{CD}$ targets of several micron thickness are used. Then, the driving ion beam is generated in the

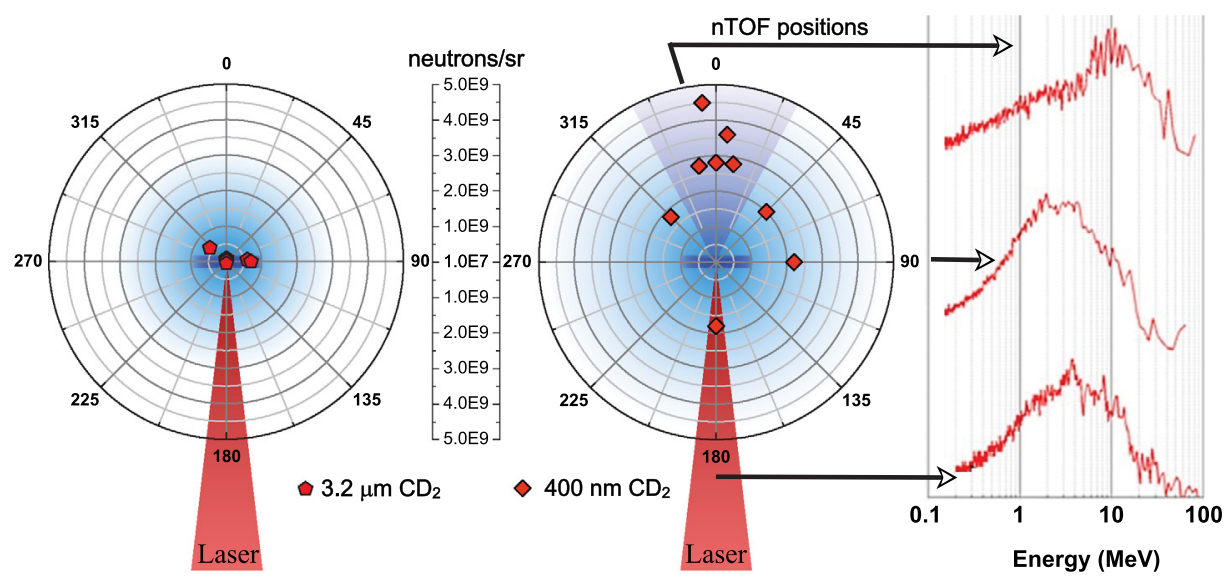

FIG. 10. Left side: Polar plot of the neutron distribution measured with bubble detectors using a $3.2 \mu \mathrm{m}$ CD driver target. The distribution is nearly isotropic. At this thickness, where TNSA is dominant, acceleration of deuterons is very inefficient and hence there is no forward component in the distribution. Right side: Polar plot of the neutron distribution from a $400 \mathrm{~nm}$ CD driver target. Two components are visible, an isotropic one from (d,n) and (p, (2)n) reactions and a strong forward directed one from deuteron break-up reactions. Far right side: Neutron spectra from nTOF-detectors at different positions around the target chamber for the $400 \mathrm{~nm} \mathrm{CD}$ shot. The neutron spectrum in forward direction (upper plot) shows the highest neutron energies, with peak flux at $15 \mathrm{MeV}$ and extending up to $60 \mathrm{MeV}$. The other detectors, middle and lower spectrum, show peak flux at a few MeV with maximum energies extending up to about $15 \mathrm{MeV}$, consistent with the results from $\mathrm{CH}$ shots. 
TNSA-regime- - the target is too thick to turn relativistically transparent-and mostly protons from the surface are accelerated. Acceleration of deuterons is suppressed and much less efficient as compared to acceleration with thinner targets in the BOA regime. This behavior is illustrated in Fig. 10. The left side shows the neutron distribution measured with bubble detectors for a $3.2 \mu \mathrm{m}$ CD target. The distribution and the neutron flux (few $10^{8} \mathrm{n} / \mathrm{sr}$ ) are comparable with shots on $\mathrm{CH}$ targets and with results presented in Ref. 25 . The right side shows a typical neutron distribution measured for a $400 \mathrm{~nm} \mathrm{CD}$ target and the corresponding neutron spectra from the nTOF-detectors at different positions (see caption of Fig. 10 for details). This forward directed distribution (in energy and flux) is advantageous for most applications as shielding requirements are reduced in comparison to $4 \pi$ distribution.

It should further be emphasized that ion bunches from typical laser-driven ion source have an initial pulse length of not more than a few picoseconds. ${ }^{6}$ Taking dispersion and the broad energy spread of the ion beam into account, the generated neutron beam will have a temporal spread of not more than a few hundred picoseconds. While up to now research utilizing neutron sources has been restricted to facilities with conventional accelerators or test reactors, this method of laser-driven neutron generation is a unique opportunity to give small scale laboratories as well as universities access to this field of research.

\section{SUMMARY}

We presented a comprehensive analysis consisted of a laser-driven neutron source. The driving ion beam consisting of either only protons from $\mathrm{CH}$ targets or protons and deuterons from CD targets with maximum energies of $80 \mathrm{MeV}$ and $100 \mathrm{MeV}$, respectively. The driving ion beam was dumped in a Be-converter for the generation of neutrons via $(p, n)$, $(\mathrm{p}, 2 \mathrm{n})$, and $(\mathrm{d}, \mathrm{n})$ reactions and via deuteron break-up reactions. The neutron beam has been characterized with a set of diagnostics consisting of a dozen bubble detectors, 3 nTOF detectors, and a neutron imager. With CD targets of $400 \mathrm{~nm}$ thickness, where efficient acceleration of deuterons takes place in the BOA regime, the neutron distribution has a strong forward directed component with $4.4 \times 10^{9} \mathrm{n} / \mathrm{sr}$ and maximum neutron energies of up to $80 \mathrm{MeV}$. This constitutes an order of magnitude higher flux and 3 to 4 times higher maximum neutron energies than previously reported. The ability to generate dense $\mathrm{MeV}$-neutron beams with lasers also opens the field of neutron and neutron-assisted research to a much broader community, including small scale laboratories as well as universities and also paves the way to new compact and cost-efficient neutron-based applications.

\section{ACKNOWLEDGMENTS}

We gratefully acknowledge the expert support of the Trident laser team and LANSCE in conducting the experiments. We thank M. Geissler and D. Green for fruitful discussions. This work was supported by LANL Laboratory Directed Research and Development (LDRD), the DOE
Office of Fusion Energy Sciences (OFES), BMBF 06Da9044I, HIC4FAIR, the University of Darmstadt, DFG, and Sandia. Sandia is a multiprogram laboratory operated by Sandia Corporation, a Lockheed Martin Company, for the National Nuclear Security Administration under Contract No. DE-AC04-94AL85000. Work was performed under the auspices of the U.S. Department of Energy by the Los Alamos National Security, LLC, Los Alamos National Laboratory.

${ }^{1}$ S. Krueger, C. W. Meuse, C. F. Majkrzak, J. A. Dura, N. F. Berk, M. Tarek, and A. L. Plant, Langmuir 17, 511 (2001).

${ }^{2}$ N. Kardjilov, F. Fiori, G. Giunta, A. Hilger, F. Rustichelli, M. Strobl, J. Banhart, and R. Triolo, J. Neutron Res. 14, 29 (2006).

${ }^{3}$ A. Buffler, Radiat. Phys. Chem. 71, 853 (2004).

${ }^{4}$ L. Perkins, B. Logan, M. Rosen, M. Perry, T. D. de la Rubia, N. Ghoniem, T. Ditmire, P. Springer, and S. Wilks, Nucl. Fusion 40, 1 (2000).

${ }^{5}$ I. Anderson, G. Bauer, D. Baxter, M. Furusaka, F. Goldenbaum, G. Mank, E. Pither, U. RosenGard, W. Wagner, and Q. Yan, "Development opportunities for small and medium scale accelerator driven neutron sources," Report of a technical meeting held in Vienna, 18-21 May 2004 (IAEA, 2005).

${ }^{6}$ S. P. Hatchett, C. G. Brown, T. E. Cowan, E. A. Henry, J. S. Johnson, M. H. Key, J. A. Koch, A. B. Langdon, B. F. Lasinski, R. W. Lee, A. J. Mackinnon, D. M. Pennington, M. D. Perry, T. W. Phillips, M. Roth, T. C. Sangster, M. S. Singh, R. A. Snavely, M. A. Stoyer, S. C. Wilks, and K. Yasuike, Phys. Plasmas 7, 2076 (2000).

${ }^{7}$ R. A. Snavely, M. H. Key, S. P. Hatchett, T. E. Cowan, M. Roth, T. W. Phillips, M. A. Stoyer, E. A. Henry, T. C. Sangster, M. S. Singh, S. C. Wilks, A. MacKinnon, A. Offenberger, D. M. Pennington, K. Yasuike, A. B. Langdon, B. F. Lasinski, J. Johnson, M. D. Perry, and E. M. Campbell, Phys. Rev. Lett. 85, 2945 (2000).

${ }^{8}$ S. C. Wilks, A. B. Langdon, T. E. Cowan, M. Roth, M. Singh, S. Hatchett, M. H. Key, D. Pennington, A. MacKinnon, and R. A. Snavely, Phys. Plasmas 8, 542 (2001).

${ }^{9}$ M. Borghesi, A. J. Mackinnon, D. H. Campbell, D. G. Hicks, S. Kar, P. K. Patel, D. Price, L. Romagnani, A. Schiavi, and O. Willi, Phys. Rev. Lett. 92, 055003 (2004).

${ }^{10}$ J. Fuchs, P. Antici, E. d'Humieres, E. Lefebvre, M. Borghesi, E. Brambrink, C. A. Cecchetti, M. Kaluza, V. Malka, M. Manclossi, S. Meyroneinc, P. Mora, J. Schreiber, T. Toncian, H. Pepin, and P. Audebert, Nat. Phys. 2, 48 (2006).

${ }^{11}$ B. M. Hegelich, B. J. Albright, J. Cobble, K. Flippo, S. Letzring, M. Paffett, H. Ruhl, J. Schreiber, R. K. Schulze, and J. C. Fernández, Nature 439, 441 (2006).

${ }^{12}$ L. Robson, P. T. Simpson, R. J. Clarke, K. W. D. Ledingham, F. Lindau, O. Lundh, T. McCanny, P. Mora, D. Neely, C.-G. Wahlstrom, M. Zepf, and P. McKenna, Nat. Phys. 3, 58 (2007).

${ }^{13}$ M. Hegelich, S. Karsch, G. Pretzler, D. Habs, K. Witte, W. Guenther, M. Allen, A. Blazevic, J. Fuchs, J. C. Gauthier, M. Geissel, P. Audebert, T. Cowan, and M. Roth, Phys. Rev. Lett. 89, 085002 (2002).

${ }^{14}$ T. E. Cowan, J. Fuchs, H. Ruhl, A. Kemp, P. Audebert, M. Roth, R. Stephens, I. Barton, A. Blazevic, E. Brambrink, J. Cobble, J. Fernández, J.-C. Gauthier, M. Geissel, M. Hegelich, J. Kaae, S. Karsch, G. P. Le Sage, S. Letzring, M. Manclossi, S. Meyroneinc, A. Newkirk, H. Pépin, and N. Renard-LeGalloudec, Phys. Rev. Lett. 92, 204801 (2004).

${ }^{15}$ T. Bartal, M. E. Foord, C. Bellei, M. H. Key, K. A. Flippo, S. A. Gaillard, D. T. Offermann, P. K. Patel, L. C. Jarrott, D. P. Higginson, M. Roth, A. Otten, D. Kraus, R. B. Stephens, H. S. McLean, E. M. Giraldez, M. S. Wei, D. C. Gautier, and F. N. Beg, Nat. Phys. 8, 139 (2012).

${ }^{16}$ S. Palaniyappan, B. M. Hegelich, H.-C. Wu, D. Jung, D. C. Gautier, L. Yin, B. J. Albright, R. P. Johnson, T. Shimada, S. Letzring, D. T. Offermann, J. Ren, C. Huang, R. Hörlein, B. Dromey, J. C. Fernandez, and R. C. Shah, Nat. Phys. 8, 763 (2012).

${ }^{17}$ L. Yin, B. J. Albright, B. M. Hegelich, and J. C. Fernández, Laser Part. Beams 24, 291 (2006).

${ }^{18}$ L. Yin, B. J. Albright, B. M. Hegelich, K. J. Bowers, K. A. Flippo, T. J. T. Kwan, and J. C. Fernandez, Phys. Plasmas 14, 056706 (2007).

${ }^{19}$ D. Jung, L. Yin, B. J. Albright, D. C. Gautier, S. Letzring, B. Dromey, M. Yeung, R. Hörlein, R. Shah, S. Palaniyappan, K. Allinger, J. Schreiber, K. 
J. Bowers, H.-C. Wu, J. C. Fernandez, D. Habs, and B. M. Hegelich, New J. Phys. 15, 023007 (2013).

${ }^{20}$ B. Hegelich, D. Jung, B. Albright, J. Fernandez, D. Gautier, C. Huang, T. Kwan, S. Letzring, S. Palaniyappan, R. Shah, H.-C. Wu, L. Yin, A. Henig, R. Hörlein, D. Kiefer, J. Schreiber, X. Yan, T. Tajima, D. Habs, B. Dromey, and J. Honrubia, Nucl. Fusion 51, 083011 (2011).

${ }^{21}$ P. A. Norreys, A. P. Fews, F. N. Beg, A. R. Bell, A. E. Dangor, P. Lee, M. B. Nelson, H. Schmidt, M. Tatarakis, and M. D. Cable, Plasma Phys. Controlled Fusion 40, 175 (1998).

${ }^{22}$ K. L. Lancaster, S. Karsch, H. Habara, F. N. Beg, E. L. Clark, R. Freeman, M. H. Key, J. A. King, R. Kodama, K. Krushelnick, K. W. D. Ledingham, P. McKenna, C. D. Murphy, P. A. Norreys, R. Stephens, C. Stöeckl, Y. Toyama, M. S. Wei, and M. Zepf, Phys. Plasmas 11, 3404 (2004).

${ }^{23}$ D. P. Higginson, J. M. McNaney, D. C. Swift, T. Bartal, D. S. Hey, R. Kodama, S. L. Pape, A. Mackinnon, D. Mariscal, H. Nakamura, N. Nakanii, K. A. Tanaka, and F. N. Beg, Phys. Plasmas 17, 100701 (2010).

${ }^{24}$ G. M. Petrov, D. P. Higginson, J. Davis, T. B. Petrova, J. M. McNaney, C. McGuffey, B. Qiao, and F. N. Beg, Phys. Plasmas 19, 093106 (2012).

${ }^{25}$ D. P. Higginson, J. M. McNaney, D. C. Swift, G. M. Petrov, J. Davis, J. A. Frenje, L. C. Jarrott, R. Kodama, K. L. Lancaster, A. J. Mackinnon, H. Nakamura, P. K. Patel, G. Tynan, and F. N. Beg, Phys. Plasmas 18, 100703 (2011).

${ }^{26}$ S. H. Batha, R. Aragonez, F. L. Archuleta, T. N. Archuleta, J. F. Benage, J. A. Cobble, J. S. Cowan, V. E. Fatherley, K. A. Flippo, D. C. Gautier, R. P. Gonzales, S. R. Greenfield, B. M. Hegelich, T. R. Hurry, R. P. Johnson, J. L. Kline, S. A. Letzring, E. N. Loomis, F. E. Lopez, S. N. Luo, D. S. Montgomery, J. A. Oertel, D. L. Paisley, S. M. Reid, P. G. Sanchez, A. Seifter, T. Shimada, and J. B. Workman, Rev. Sci. Instrum. 79, 10F305 (2008).

${ }^{27}$ L. Yin, B. J. Albright, K. J. Bowers, D. Jung, J. C. Fernández, and B. M. Hegelich, Phys. Rev. Lett. 107, 045003 (2011).

${ }^{28}$ D. Jung, R. Hörlein, D. C. Gautier, S. Letzring, D. Kiefer, K. Allinger, B. J. Albright, R. Shah, S. Palaniyappan, L. Yin, J. C. Fernández, D. Habs, and B. M. Hegelich, Rev. Sci. Instrum. 82, 043301 (2011).
${ }^{29}$ D. Jung, R. Horlein, D. Kiefer, S. Letzring, D. C. Gautier, U. Schramm, C. Hubsch, R. Ohm, B. J. Albright, J. C. Fernandez, D. Habs, and B. M. Hegelich, Rev. Sci. Instrum. 82, 013306 (2011).

${ }^{30}$ Y. Rao, A. Davis, T. Spencer, and R. Filz, Nucl. Instrum. Methods 180, 153 (1981)

${ }^{31}$ G. Rusch, E. Winkel, A. Noll, and W. Heinrich, Int. J. Rad. Appl. Instrum. D 19, 261 (1991).

${ }^{32}$ I. J. Paterson, R. J. Clarke, N. C. Woolsey, and G. Gregori, Meas. Sci. Technol. 19, 095301 (2008).

${ }^{33}$ M. M. Günther, J. Schütrumpf, A. Britz, K. Vogt, K. Sonnabend, and M. Roth, Fusion Sci. Technol. 61, 231 (2012).

${ }^{34}$ F. Nurnberg, M. Schollmeier, E. Brambrink, A. Blazevic, D. C. Carroll, K. Flippo, D. C. Gautier, M. Geissel, K. Harres, B. M. Hegelich, O. Lundh, K. Markey, P. McKenna, D. Neely, J. Schreiber, and M. Roth, Rev. Sci. Instrum. 80, 033301 (2009).

${ }^{35}$ G. W. Cooper and C. L. Ruiz, Rev. Sci. Instrum. 72, 814 (2001).

${ }^{36} \mathrm{See} \mathrm{http://www.bubbletech.ca} \mathrm{for} \mathrm{BTI,} \mathrm{Bubble} \mathrm{Technology} \mathrm{Industries.}$

${ }^{37}$ R. H. Olsher, T. D. McLean, M. W. Mallett, L. L. Romero, R. T. Devine, and J. M. Hoffman, Radiat. Prot. Dosim. 126, 326 (2007).

${ }^{38}$ F. Smecka and M. Hajek, "Neutronendosimetrie mit bubbledetektoren," AIAU Paper No. 2007-27607, Technische Universität Wien, 2007.

${ }^{39}$ G. P. Grim, G. L. Morgan, M. D. Wilke, P. L. Gobby, C. R. Christensen, and D. C. Wilson, Rev. Sci. Instrum. 75, 3572 (2004).

${ }^{40}$ C. R. Christensen, C. W. Barnes, G. L. Morgan, M. Wilke, and D. C. Wilson, Rev. Sci. Instrum. 74, 2690 (2003).

${ }^{41}$ L. Yin, B. J. Albright, D. Jung, R. C. Shah, S. Palaniyappan, K. J. Bowers, A. Henig, J. C. Fernandez, and B. M. Hegelich, Phys. Plasmas 18, 063103 (2011).

${ }^{42}$ C. G. Freeman, G. Fiksel, C. Stoeckl, N. Sinenian, M. J. Canfield, G. B. Graeper, A. T. Lombardo, C. R. Stillman, S. J. Padalino, C. Mileham, T. C. Sangster, and J. A. Frenje, Rev. Sci. Instrum. 82, 073301 (2011).

${ }^{43}$ A. Mancic, J. Fuchs, P. Antici, S. A. Gaillard, and P. Audebert, Rev. Sci. Instrum. 79, 073301 (2008).

${ }^{44}$ N. E. Davison, M. J. Canty, D. A. Dohan, and A. McDonald, Phys. Rev. C 10, 50 (1974).

${ }^{45}$ A. De, S. Ray, and S. K. Ghosh, Phys. Rev. C 37, 2441 (1988). 